\title{
Non-vitamin K oral antagonist failure and tailored treatment in patients with atrial fibrillation and stroke
}

\author{
Hyung Jun Kim ${ }^{1}$, Soyoun Choi ${ }^{1}$, Hee-Jin Kim ${ }^{2}$, Oh Young Bang ${ }^{1}$ \\ ${ }^{1}$ Department of Neurology, Samsung Medical Center, Sungkyunkwan University School of Medicine, Seoul, Korea \\ ${ }^{2}$ Department of Laboratory Medicine and Genetics, Samsung Medical Center, Sungkyunkwan University School of Medicine, \\ Seoul, Korea
}

Received: February 11, 2020

Revised: March 1, 2020

Accepted: March 2, 2020

Corresponding author:

Oh Young Bang

Department of Neurology,

Samsung Medical Center,

Sungkyunkwan University

School of Medicine, 81 Irwon-

ro, Gangnam-gu, Seoul 06351 , Korea

Tel: +82-2-3410-2114

E-mail:

ohyoung.bang@samsung.com

\begin{abstract}
In case of any embolic event, treatment strategy should be established according to the pathomechanisms of failure of non-vitamin K oral anticoagulants (NOAC). A 72-year-old man was referred to our hospital with a sudden speech disturbance. Diffusion-weighted imaging revealed cerebral infarction in the left middle cerebral artery territory. The patient had been diagnosed with nonvalvular atrial fibrillation 3 years ago and was administered a dose of $60 \mathrm{mg}$ edoxaban/day. Anti-Xa and factor Xa activity assay confirmed the effect of this drug. A detailed transthoracic and transesophageal echocardiography revealed fibroelastoma of mitral valve, atrial septal defect, and spontaneous echo contrast in the left atrial appendage. Although the drug was effective, cardioembolism occurred and hence we decided to perform surgery. As the etiology of NOAC failure varies, anti-Xa and factor Xa activity assays help to determine the cause modifying the treatment strategy accordingly.
\end{abstract}

Keywords: Atrial fibrillation; Cerebral infarction; Edoxaban; Factor Xa

\section{INTRODUCTION}

Oral anticoagulants (OACs) help to prevent stroke or systemic embolism (SE) in patients diagnosed with atrial fibrillation (Afib) [1-4]. Although the effects of vitamin $\mathrm{K}$ antagonist (VKA) and non-vitamin $\mathrm{K}$ oral anticoagulants (NOAC) are superior to the antiplatelet agents in prevention of strokes in these patients, upto one-fifth to one-third of them still experience stroke/SE after taking them. Although we may consider switching OAC (e.g., VKA to NOAC or NOAC to other

This is an Open Access article distributed under the terms of the Creative Commons Attribution Non-Commercial License (https:// creativecommons.org/licenses/ by-nc/4.0/).
NOACs) or combining them with antiplatelet agents in later cases of patients, randomized trials are unable to provide any evidence supporting the treatment and pathomechanisms related to failure of NOAC.

We reported a case of an Afib patient who had cardioembolic stroke although he had good 
compliance with edoxaban. We discussed the possible mechanisms of NOAC failure in his case.

\section{CASE REPORT}

A 72-year-old man showed inability to speak and was referred to our hospital. He had been diagnosed with persistent AFib 3 years ago and also had a history of diabetes as well as hypertension. The patient was administered $60 \mathrm{mg}$ of edoxaban for the same $\left(\mathrm{CHA}_{2} \mathrm{DS}_{2}-\mathrm{VASc}\right.$ score $\left.=3\right)$. He had been taking this medication regularly and did not show any side effects or thromboembolic episodes. Although his cranial motor and sensory exams were normal, transcortical motor aphasia revealed decreased spontaneous speech, naming, and writing abilities. It was observed that he had intact repetition and comprehension skills.
Although results of all the routine laboratory tests were normal, the coagulation test revealed prothrombin time (PT) of $16.0 \mathrm{sec}$ when the normal range was between 12.6 to $14.9 \mathrm{sec}-$ onds and international normalized ratio (INR) was 1.27 when normal range was between 0.90 to 1.10 . Diffusion-weighted imaging (DWI) that was performed on the admission day revealed cortical-subcortical infarcts in the left middle cerebral artery territory (Fig. 1A, B). Magnetic resonance angiography (MRA) did not reveal artery stenosis (Fig. 1C, D). To confirm the efficacy of edoxaban, anti-Xa and factor Xa activity assays were performed. Blood samples were taken just before the administration of the next medication. The anti-Xa activity was observed to be increased in the blood $[5,6]$ as the trough value of $158.21 \mathrm{ng} / \mathrm{mL}$ was observed to be higher than the mean trough value that was observed in the previous study (104 ng/mL). Additionally factor Xa activity was found to be
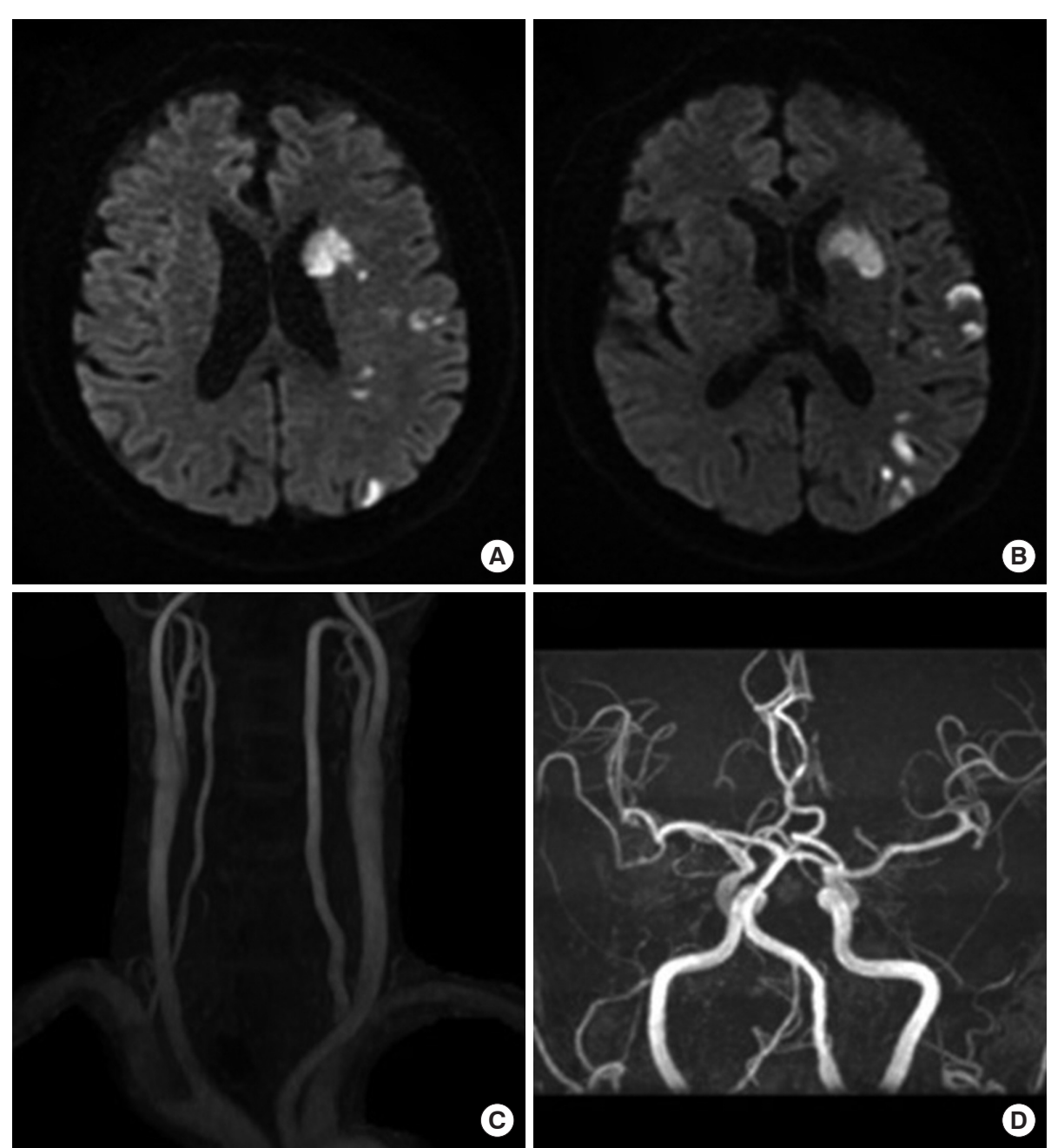

Fig. 1. (A, B) Brain magnetic resonance imaging (MRI) reveal acute scattered cerebral infarctions in the middle cerebral artery territory with involvement of the putamen, globus pallidus, temporal, and parietal cortical-subcortical areas. (C, D) Magnetic resonance angiography show a no relevant stenosis of intra-extracranial vessels. 
decreased by $61 \%$ compared with its average baseline endogenous activity $(92.1 \% \pm 20.9 \%$, relative to normal control samples) [7]. Detailed investigations further analyzed the cardiac functions to find the possible causes of cardioembolism besides AFib. Electrocardiogram (ECG) showed persistent Afib rhythm and normal heart rate (60 to $80 / \mathrm{min}$ ). TTE revealed normal regional wall motion, normal left ventricular systolic function (ejection fraction $=69 \%$ ), and diastolic dysfunction $\left(E / e^{\prime}=16.3\right)$ of the enlarged left atrium (left atrium volume index was $123.11 \mathrm{~mL} / \mathrm{m}^{2}$ ). Additionally long axis (Fig. $2 \mathrm{~A})$ and four-chambered view revealed lesion of the mitral valve. A round mass of $0.44 \times 0.41 \mathrm{~cm}$ (A2 portion) was observed in anterior mitral valve leaflet (Fig. 2B), spontaneous echo contrast (SEC) of left atrial appendage (LAA) (Fig. 2D), and atrial septal defect (ASD) (Fig. 2C).

After the discussion with neurologists, cardiologists, and cardiac surgeons, the patient underwent resection of cardiac mass, primary ASD closure, and modified Cox-Maze III operation utilizing cryoablation. Post-operation, ECG revealed a sinus rhythm. TTE did not reveal any cardiac mass or ASD flow. Pathological examination identified the cardiac mass as fibroelastoma. Rivaroxaban $20 \mathrm{mg}$ is administered after surgery and changes to antiplatelet are considered after 3 months. Recurrence of stroke was not observed in these patients postoperatively.

Written informed consent was obtained from the patient for the publication of this case report.
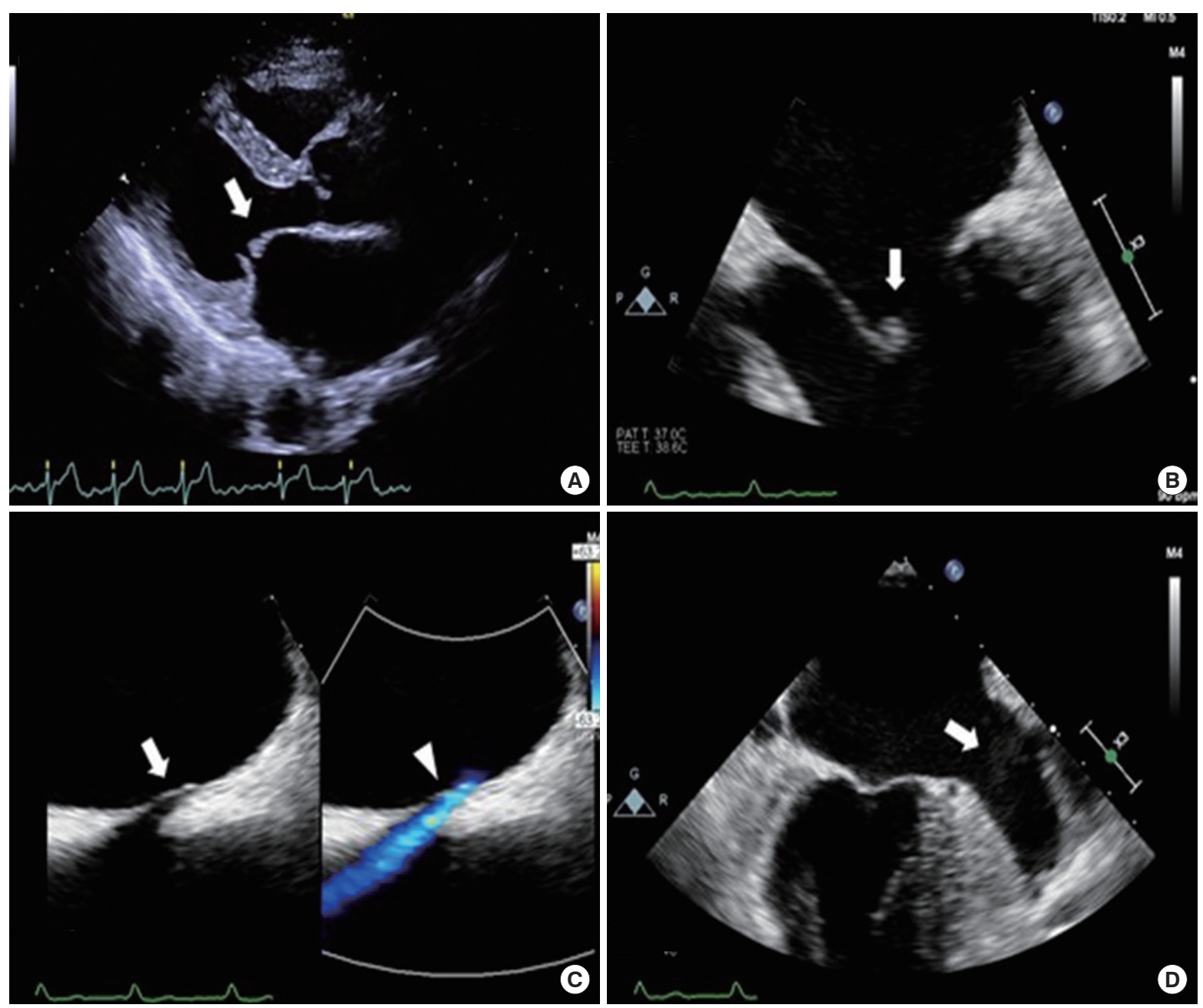

Fig. 2. Transesophageal echocardiography (TEE). The long axis (A) view shows hyperechogenic mass (white arrow) that is attached to atrial side of $A 2$ scallop of the anterior mitral leaflet. (B) The four-chamber view at $0^{\circ}$ shows a mobile nodular mass (white arrow), measuring 0.44 $\times 0.41 \mathrm{~cm}$, on the anterior leaflet of the mitral valve. (C) The color doppler echocardiograpy shows a secundum defect (white arrow) with left-to-right shunt in the middle of atrial septum (arrowhead). (D) The midesophageal view of left atrial appendage demonstrates spontaneous echo contrast (white arrow). 


\section{DISCUSSION}

Pivotal randomized clinical trials demonstrated that NOACs are superior and effective to VKAs in the patients diagnosed with Afib leading to significantly lower rates of hemorrhage and death from cardiovascular causes [1-4]. These drugs are prescribed to the patients to prevent strokes as food-drug interactions are not observed and they are not require routine therapeutic monitoring. Strokes/SEs were observed in patients taking NOACs. ENGAGE AF TIMI 48 trial was conducted in 21,104 patients diagnosed with moderate-to-severe Afib. It was observed that the annual rate of stroke/SE was 1.18\% when high dose edoxaban was administered and $1.61 \%$ when low-dose edoxaban was administered $(1.50 \%$ with VKA) [4].

Failure of the antithrombotic therapy can be as a result of resistance to these drugs, incorrect diagnosis, uncontrolled risk factors, poor absorption, and drug interactions. First, it would be reasonable to classify failure of therapy in the patients who have stroke even when they are administered with NOACs because they are poor compliance and/or intolerant to them. The incidence of an acute ischemic stroke 60 days preceding the withdrawal of antiplatelet antithrombotic medications was associated with $5.2 \%$ of all the ischemic strokes (47.5\% in the periprocedural period) [8] and the same may be true for patients with Afib-related stroke. However, the patient had good compliance to the medication prior to the stroke. Secondly, NOACs can be less effective if they are administered in lower doses, the patient is unable to absorb them, and they interact with other drugs. A recent study included 713 Afib patients who had acute ischemic stroke and who were administered NOAC. Two-thirds of these patients had cardioembolic pathogenesis, atrial enlargement, and high $\mathrm{CHA}_{2} \mathrm{DS}_{2}$-VASC scores that were associated with an increased risk of stroke [9]. Patients often take anti-arrhythmic drugs and antibiotics that influence the levels and interactions of NOAC [10]. Although the effect of these medicines on NOAC need to be considered, our patient was not taking any.

Thirdly, coagulation tests or blood levels of NOACs should be conducted on the patients who have had recurrent stroke/ $\mathrm{SE}$. A retrospective study showed that the use of NOAC was associated with a higher risk of ischemic stroke than with VKA and suggested a prescribed dose of this medicine [11]. Several coagulation tests provide information on the prolonged activated partial thromboplastin time (aPPT) in response to the level of dabigatran and prolonged PT in response to the level of rivaroxaban [12]. Prolonged PT was observed in our patient suggesting that edoxaban was effective. Although the aPPT and PT/INR can demonstrate the efficacy of edoxaban, they have low sensitivity. These tests may not exclude clinically relevant on-therapy drug levels [13]. In contrast, anti-Xa factor Xa activity assays predict concentration and efficacy of edoxaban. Although the previous studies reported coefficient of determination $\left(R^{2}\right)$ values of PT/INR and aPTT as 0.95 , the $R^{2}$ value of anti-Xa activity exceeded 0.95 [13]. Anti-Xa activity assay quantified edoxaban as a pharmacokinetic marker and showed a positive linear relationship to the blood drug level $[6,13]$. If the anti-Xa activity increases sufficiently after the administration of edoxaban, the endogenous factor Xa activity is inhibited. This reduced factor Xa activity inhibits the coagulation pathway and anticoagulation occurs when prothrombin is converted to thrombin. Therefore, the factor Xa activity is a pharmacodynamics marker that can measure edoxaban efficacy [7]. Although the patient was administered an adequate dose of this drug and had good compliance, he still had a thromboembolic episode and therefore these assays were conducted to check its efficacy. It was observed that the activity of anti-Xa activity increased and the factor Xa was sufficiently inhibited, suggesting an anticoagulating function of the drug on this patient. Although recently point-of-care testing was introduced to measure the blood rivaroxaban level in these patients, further tests are still needed to guide their decision regarding thrombolytic therapy [14-16].

Strokes unrelated to Afib are another cause of NOAC failure in these patients. Compared with OAC, the rate of stokes increase in the patients treated with antiplatelets. The incidences of strokes that occur in one third of the patients are unrelated to Afib. The incidence of strokes in one in every seven patients had a non-Afib pathogenesis [17]. The infarct pattern revealed by the DWI was correlated with its underlying pathogenic mechanisms [18]. MRA revealed an embolic stroke and the absence of any arterial lesion that precluded the possibility that it was cardioembolic stroke. Transesophageal echocardiography revealed the presence of cardioembolic infarction that occurred in relation to the fibroelastoma of mitral valve [19], ASD [20], and SEC of LAA [21]. These factors increased the risk of cardiogenic embolism. Due to the effect of this drug, thrombus was rapidly recanalized preventing an occlusion resulting in the formation of scattered infarction and neurologic deficit that was found to be insignificant.

Poorly controlled risk factors multiply the occurrence of stroke in patients diagnosed with Afib, regardless of the use 
of OACs [9]. Therefore, it is important to adequately control the heart rate and prevent other comorbidities such as heart failure, hypertension, and diabetes that are modifiable components of $\mathrm{CHA}_{2} \mathrm{DS}_{2}$-VASc score and are closely associated with risk of stroke/SE.

Although the baseline factor Xa activity of our patients could not be known, the patient had good compliance, the anti-Xa activity was sufficiently increased, and the factor Xa activity was lower than the average baseline endogenous activity [7], indicating that the drug was effective. Despite sufficient edoxaban effects, a cardioembolic infarction occurred in our patient relation to the fibroelastoma of mitral valve, ASD, and SEC of LAA, which were associated with an increased cardiogenic embolic risk. Unlike NOACs are contraindication in valvular Afib such as mitral stenosis, no guideline has been suggested in relation to these structural heart disease. Therefore, surgical treatment was chosen rather than other NOAC or VKA.

Our present case suggests that if thromboembolism occurs while taking edoxaban, anti-Xa and factor Xa activity assays can be used to determine the effects of drugs and a treatment strategy can be modified accordingly.

\section{CONFLICTS OF INTEREST}

No potential conflict of interest relevant to this article was reported.

\section{ORCID}

Hyung Jun Kim

https://orcid.org/0000-0002-7518-9739

Soyoun Choi

Hee-Jin Kim https://orcid.org/0000-0002-9581-778X

Oh Young Bang

https://orcid.org/0000-0003-3741-4613

https://orcid.org/0000-0002-7962-8751

\section{AUTHOR CONTRIBUTIONS}

Conception or design: HJK, HJK, OYB.

Acquisition, analysis, or interpretation of data: HJK, SC, HJK, OYB.

Drafting the work or revising: HJK, OYB.

Final approval of the manuscript: OYB.

\section{REFERENCES}

1. Connolly SJ, Ezekowitz MD, Yusuf S, Eikelboom J, Oldgren J, Parekh A, et al. Dabigatran versus warfarin in pa- tients with atrial fibrillation. N Engl J Med 2009;361:113951.

2. Patel MR, Mahaffey KW, Garg J, Pan G, Singer DE, Hacke W, et al. Rivaroxaban versus warfarin in nonvalvular atrial fibrillation. N Engl J Med 2011;365:883-91.

3. Granger CB, Alexander JH, McMurray JJ, Lopes RD, Hylek EM, Hanna M, et al. Apixaban versus warfarin in patients with atrial fibrillation. N Engl J Med 2011;365:981-92.

4. Giugliano RP, Ruff CT, Braunwald E, Murphy SA, Wiviott $\mathrm{SD}$, Halperin JL, et al. Edoxaban versus warfarin in patients with atrial fibrillation. N Engl J Med 2013;369:2093104.

5. Shin H, Cho MC, Kim RB, Kim CH, Choi NC, Kim SK, et al. Laboratory measurement of apixaban using anti-factor Xa assays in acute ischemic stroke patients with non-valvular atrial fibrillation. J Thromb Thrombolysis 2018;45:250-6.

6. Ruff CT, Giugliano RP, Braunwald E, Morrow DA, Murphy SA, Kuder JF, et al. Association between edoxaban dose, concentration, anti-factor Xa activity, and outcomes: an analysis of data from the randomised, double-blind ENGAGE AF-TIMI 48 trial. Lancet 2015;385:2288-95.

7. Yin OQP, Antman EM, Braunwald E, Mercuri MF, Miller R, Morrow $\mathrm{D}$, et al. Linking endogenous factor Xa activity, a biologically relevant pharmacodynamic marker, to edoxaban plasma concentrations and clinical outcomes in the ENGAGE AF-TIMI 48 trial. Circulation 2018;138:1963-73.

8. Broderick JP, Bonomo JB, Kissela BM, Khoury JC, Moomaw CJ, Alwell K, et al. Withdrawal of antithrombotic agents and its impact on ischemic stroke occurrence. Stroke 2011; 42:2509-14.

9. Paciaroni M, Agnelli G, Caso V, Silvestrelli G, Seiffge DJ, Engelter $\mathrm{S}$, et al. Causes and risk factors of cerebral ischemic events in patients with atrial fibrillation treated with nonvitamin $\mathrm{K}$ antagonist oral anticoagulants for stroke prevention. Stroke 2019;50:2168-74.

10. Steffel J, Verhamme P, Potpara TS, Albaladejo P, Antz M, Desteghe L, et al. The 2018 European Heart Rhythm Association Practical Guide on the use of non-vitamin Kantagonist oral anticoagulants in patients with atrial fibrillation. Eur Heart J 2018;39:1330-93.

11. Shpak M, Ramakrishnan A, Nadasdy Z, Cowperthwaite M, Fanale $\mathrm{C}$. Higher incidence of ischemic stroke in patients taking novel oral anticoagulants. Stroke 2018;49:2851-6.

12. Weitz JI, Eikelboom JW. Urgent need to measure effects of direct oral anticoagulants. Circulation 2016;134:186-8.

13. Cuker A, Husseinzadeh H. Laboratory measurement of the anticoagulant activity of edoxaban: a systematic re- 
view. J Thromb Thrombolysis 2015;39:288-94.

14. Ebner M, Peter A, Spencer C, Hartig F, Birschmann I, Kuhn $\mathrm{J}$, et al. Point-of-care testing of coagulation in patients treated with non-vitamin $\mathrm{k}$ antagonist oral anticoagulants. Stroke 2015;46:2741-7.

15. Ebner M, Birschmann I, Peter A, Spencer C, Hartig F, Kuhn $\mathrm{J}$, et al. Point-of-care testing for emergency assessment of coagulation in patients treated with direct oral anticoagulants. Crit Care 2017;21:32.

16. Seiffge DJ, Kagi G, Michel P, Fischer U, Bejot Y, Wegener S, et al. Rivaroxaban plasma levels in acute ischemic stroke and intracerebral hemorrhage. Ann Neurol 2018;83:4519.

17. Kim SJ, Ryoo S, Kwon S, Park YK, Kim JP, Lee GY, et al. Is atrial fibrillation always a culprit of stroke in patients with atrial fibrillation plus stroke? Cerebrovasc Dis 2013;36:373-
82.

18. Bang OY, Li W. Applications of diffusion-weighted imaging in diagnosis, evaluation, and treatment of acute ischemic stroke. Precis Future Med 2019;3:69-76.

19. Brown RD Jr, Khandheria BK, Edwards WD. Cardiac papillary fibroelastoma: a treatable cause of transient ischemic attack and ischemic stroke detected by transesophageal echocardiography. Mayo Clin Proc 1995;70:863-8.

20. Mandalenakis Z, Rosengren A, Lappas G, Eriksson P, Hansson PO, Dellborg M. Ischemic stroke in children and young adults with congenital heart disease. J Am Heart Assoc 2016;5:e003071.

21. Zhao Y, Ji L, Liu J, Wu J, Wang Y, Shen S, et al. Intensity of left atrial spontaneous echo contrast as a correlate for stroke risk stratification in patients with nonvalvular atrial fibrillation. Sci Rep 2016;6:27650. 